\begin{tabular}{|c|c|c|c|}
\hline \multirow{2}{*}{$\begin{array}{r}\text { Case Reports in } \\
\text { Gastroenterology }\end{array}$} & \multicolumn{2}{|c|}{ Case Rep Gastroenterol 2018;12:41-45 } & \multirow[b]{2}{*}{$\begin{array}{l}\text { Karger } \\
\text { Openaccess }\end{array}$} \\
\hline & $\begin{array}{l}\text { DOI: } 101159 / 000486192 \\
\text { Published online: February 1, } 2018\end{array}$ & $\begin{array}{l}\text { (c) } 2018 \text { The Author(s) } \\
\text { Published by S. Karger AG, Basel } \\
\text { www.karger.com/crg }\end{array}$ & \\
\hline & $\begin{array}{l}\text { This article is licensed under the } \\
\text { International License (CC BY-NC) } \\
\text { Usage and distribution for commer }\end{array}$ & $\begin{array}{l}\text { nons Attribution-NonCommercial } 4 . \\
\text { ger.com/Services/OpenAccessLicense) } \\
\text { uires written permission. }\end{array}$ & \\
\hline
\end{tabular}

\title{
Sevelamer-Induced Gastrointestinal Injury Presenting as Gastroenteritis
}

\author{
Jared Magee Matthew Robles Peter Dunaway \\ Madigan Army Medical Center, Tacoma, WA, USA
}

\section{Keywords}

Sevelamer $\cdot$ Crystal-induced $\cdot$ Gastroenteritis

\begin{abstract}
End-stage renal disease (ESRD) is a common disease encountered in clinical practice and is associated with increasing metabolic derangements through disease progression. Phosphate retention is one of the most common derangements and is associated with increased mortality. Hyperphosphatemia becomes increasingly prevalent as glomerular filtration rate decreases. Non-calcium phosphate-binding resins and dietary phosphate restriction are the mainstays for managing hyperphosphatemia in patients with ESRD. Sevelamer carbonate is the most frequently used non-calcium phosphate binder in the US due to ease of administration and an excellent safety profile. However, there is increasing recognition of gastrointestinal injury associated with medication use. Here we report a case of an 81-year-old male with biopsy-proven gastrointestinal mucosal injury secondary to sevelamer carbonate administration.

C 2018 The Author(s)

Published by S. Karger AG, Basel
\end{abstract}

\section{Introduction}

End-stage renal disease (ESRD) is a frequently encountered disease with estimated prevalence of disease of 2,067 per million in the United States [1]. Cardiovascular disease is the leading cause of death within this population [2]. Observational studies have shown an association between cardiovascular mortality and increases in phosphate and calcium phosphate product [3]. Managing metabolic derangements in the setting of ESRD is a frequent issue encountered in practice, with hyperphosphatemia among the most common abnormalities encountered. 
Sevelamer is an anion exchange resin used to manage hyperphosphatemia in ESRD. Initially approved by the United States Food and Drug Administration in October 1998 as sevelamer hydrochloride (Renagel $\odot$ ), it has been largely replaced by sevelamer carbonate (Renvela ${ }^{\odot}$ ) since 2007. While both preparations show similar efficacy, Renvela is attributed with less risk of metabolic acidosis [4]. Renvela is composed of a non-absorbed hydrogel with ammonia $\left(\mathrm{NH}_{3}\right)$ on the carbonate. In the acid milieu of the stomach, the polymer dissociates from its anion and is protonated to ammonium $\left(\mathrm{NH}_{4}+\right)$. Once ammonium is formed, it is able to bind phosphate $\left(\mathrm{PO}_{4}^{-}\right)$within the intestine [5]. Though nausea, vomiting, and diarrhea are listed side effects of sevelamer, there is little information documenting gastroenteritis with sevelamer as the cause. Here we describe a case of sevelamer-induced gastrointestinal injury presenting as gastroenteritis.

\section{Case Presentation}

An 81-year-old man with a history of ESRD and hypertension presented to our hospital for profuse watery diarrhea, non-bloody emesis, abdominal pain, and subjective fever to $104.3^{\circ} \mathrm{F}$ of 1 day duration. At the time of admission, his physical exam was absence of pain or distention. Stool culture and polymerase chain reaction (PCR) for Clostridium difficile were collected, computed topography (CT) of abdomen/pelvis was performed, and rehydration with $2 \mathrm{~L}$ normal saline was given. Stool cultures and PCR were negative for $C$. difficile. CT revealed bowel wall thickening involving the duodenum and proximal jejunum consistent with duodenitis and enteritis. He improved after 4 days of conservative management of rehydration with a total of $5 \mathrm{~L}$ normal saline and antiemetic therapy with Zofran. He was discharged home with presumed viral gastroenteritis.

He returned to our hospital 4 days later for continued watery diarrhea and non-bloody emesis since time of discharge. An extended workup to include serum $B_{12}(789 \mathrm{pg} / \mathrm{mL})$, folate $(>20.0 \mathrm{ng} / \mathrm{mL})$, serum iron $(36 \mu \mathrm{g} / \mathrm{dL})$, transferrin $(147 \mathrm{mg} / \mathrm{dL})$, iron-binding capacity $(210 \mu \mathrm{g} / \mathrm{dL})$, iron saturation (17\%), ferritin (497 ng/mL), serum gliadin Ab IgA (0.2 ELISA units $/ \mathrm{mL}$ ), tissue transglutaminase Ab IgA (0.2 ELISA units $/ \mathrm{mL}$ ), erythrocyte sedimentation rate $(55 \mathrm{~mm} / \mathrm{h}), \mathrm{C}$-reactive protein $(9.1 \mathrm{mg} / \mathrm{dL})$, and stool culture (heavy normal fecal flora; negative for Campylobacter, Salmonella, Shigella, Escherichia coli 0157, EHEC, Aeromonas, Vibrio, Yersinia, and Plesiomonas), ova and parasites (negative), leukocytes (positive), and fat (slightly increased) was performed to rule out malabsorption syndrome, inflammatory condition, or infectious etiology. With the exception of fecal leukocytes, all studies were within normal limits or at the patient's baseline values compared to 1 year prior. An upper endoscopy was performed showing multiple non-obstructing duodenal ulcers oozing blood located from the bulb to the 3rd/4th portion of the duodenum. Tissue samples of ulcers were taken and sent for pathologic evaluation. Of note, the patient had not been prescribed NSAIDs and denied taking over-the-counter products prior to admission. He was started on Nexium $40 \mathrm{mg}$ twice daily and Carafate $1 \mathrm{mg}$ four times daily. He improved with these interventions and was discharged home after a 5-day hospital stay.

The patient returned to our hospital for the third time, within 3 days of discharge, with similar complaints of nausea, vomiting, fever, and excessive diarrhea that had worsened since time of discharge. However, at this time, he endorsed some bright red blood in his stool. Fecal occult blood test was performed and positive. He was started on empiric antibiotics of Levofloxacin and Flagyl $500 \mathrm{mg}$ for 5 days and received 2 units of packed red blood cells for an acute drop in his hemocrit from 31.6 at previous admission to 21.3 at current 
admission. Repeat upper endoscopy and colonoscopy were performed. Upper endoscopy was not significantly changed from prior, showing similar ulcers as above that had improved when compared to previous admission. Colonoscopy demonstrated patchy erythematous mucosa in the distal and terminal ileum. At this time, our initial pathology reports from the duodenal biopsies obtained during his previous stay resulted. Unfortunately, they were inconclusive but suggested an underlying inflammation of the intestinal mucosa. The samples were then sent to the Joint Pathology Center (JPC) for a second option. The patient was started on Prednisone $40 \mathrm{mg}$ taken once daily to address his idiopathic colitis. He improved after steroid administration and was discharged from the hospital with a 2-week supply of Prednisone $40 \mathrm{mg}$. He was instructed to follow up in our gastroenterology clinic in 1 month.

After discharge, the JPC contacted our hospital with their findings. Review of his duodenal biopsies showed basophilic coral-like nonbirefringent material with an internal "fishscale" pattern suggestive of sevelamer deposition (Fig. 1). Given the patient was actively taking sevelamer carbonate for his ESRD, we concluded the patient most likely had sevelamer-induced duodenal ulceration. The patient's sevelamer prescription was discontinued and symptoms did not return after discontinuation. He continues to receive care at our facility. Repeat EGDs and colonoscopies since discharge have demonstrated complete resolution of all findings without new areas of inflammation.

\section{Discussion}

Sevelamer-associated gastrointestinal injury presents a diagnostic challenge for clinicians and is likely an underrecognized etiology of gastrointestinal symptoms in patients with ESRD. Although adverse side effects reported in patients receiving sevelamer included vomiting, nausea, diarrhea, dyspepsia, abdominal pain, flatulence, and constipation, there has been little investigation to determine the mechanism for these symptoms [6]. These symptoms represent an important factor in compliance with therapy but are often ignored by practitioners. Sevelamer has been shown to have mucosal depositions throughout the gastrointestinal tract with varying levels of symptoms associated. Histological features of sevelamer crystals include broad, curved, and irregularly spaced "fish scales" with variable colors during in vitro and in vivo studies [7]. Our patient displayed the characteristic histopathological findings of sevelamer crystals in lesion biopsies. In the absence of diagnostic evidence of other disease process and clear resolution of symptoms after cessation of medication, we believe this to be the most plausible etiology of our patient's presentation. There is a lack of literature reporting the incidence of mucosal injury associated with crystal fragments. Identifying direct deposition of sevelamer crystals in areas of ulceration is suggestive of causation, but crystal deposition may be present in absence of symptoms or mucosal findings. There is a dose-dependent relationship with severity of lesions and sevelamer dosage but it is unclear if other factors can be attributed [7].

With the increasing utilization of sevelamer in ESRD patients, it is important to recognize potential for gastrointestinal mucosal injury. At this time, there is no known strategy for prevention or treatment of sevelamer-associated gastrointestinal injury besides avoidance of offending agent. With a seemingly low incidence rate, this is an unacceptable option for most patients with ESRD. We offer this case report to aid in broadening the differential diagnoses of physicians to include sevelamer-associated gastrointestinal injury in patients currently taking Renvela or Renagel presenting with gastroenteritis. 


\section{Acknowledgements}

Stephen C. Groo MD, Attending Physician, Department of Pathology, Madigan Army Medical Center. Michael Lewin-Smith MD, Pathologist, Department of Environment Pathology, Joint Pathology Center.

\section{Statement of Ethics}

The authors declare that there are no conflicts of interest regarding the publication of this paper.

\section{Disclosure Statement}

The views expressed are those of the authors and do not reflect the official policy or position of the US Army Medical Department, Department of the Army, Department of Defense, or the US Government.

\section{References}

1 United States Renal Data System. USRDS 2016 Annual Data Report: Atlas of Chronic Kidney Disease and End-Stage Renal Disease in the United States. National Institutes of Health; National Institute of Diabetes and Digestive and Kidney Diseases, Bethesda, MD 2016.

-2 Block GA, Hulbert-Shearon TE, Levin NW, Port FK: Association of serum phosphors and calcium x phosphate product with mortality risk in chronic hemodialysis patients: a national study. Am J Kidney Dis 1998;31:607-617.

3 Molony DA, Stephens BW: Derangements in phosphate metabolism in chronic kidney diseases/endstage renal disease: therapeutic considerations. Adv Chronic Kidney Dis 2011;18:120131.

4 Pai AB, Shepler BM: Comparison of sevelamer hydrochloride and sevelamer carbonate: risk of metabolic acidosis and clinical implications. Pharmacotherapy 2009;29:554-561.

5 Barna MM, Kapoian T, O'Mara NB: Sevlamer carbonate. Ann Pharmacother 2010;44:127-134.

6 Renagel (sevelamer hydrochloride) (product monograph). Laval, Quebec, Canada: Sanofi-Aventis Canada; August 2017.

7 Swanson B, Limketkai B, Liu T, et al: Sevelamer crystals in the gastrointestinal tract (GIT). Am J Surg Pathol 2013;37:1686-1693. 
 www.karger.com/crg \\ Magee et al.: Sevelamer-Induced Gastrointestinal Injury Presenting as Gastroenteritis}

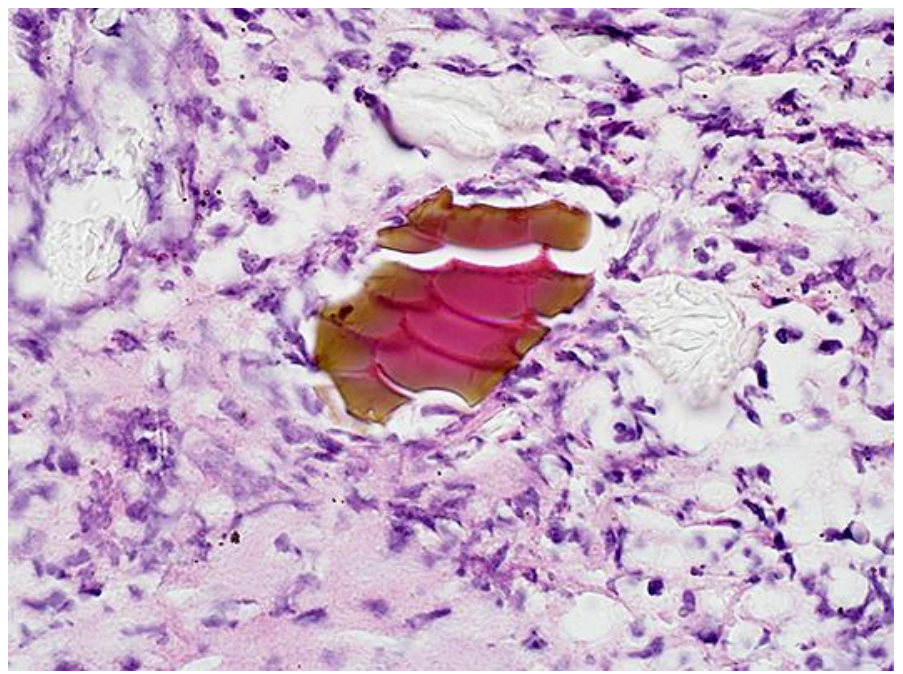

Fig. 1. Hematoxylin and eosin-stained section with nonbirefringent material displaying an internal "fishscale" pattern with rusty brown exterior and pink interior particles. The section is displayed at $60 \times$ magnification. 